Original publication:

D. Wolf and C. Kübel, Practical Metallography 55 (2018), p. 527-538

https://www.hanser-elibrary.com/doi/10.3139/147.110536

DOI: $10.3139 / 147.110536$

\title{
Electron Tomography for 3D imaging of Nanoscale Materials
}

\author{
Daniel Wolf ${ }^{1 *}$, Christian Kübel ${ }^{2}$ \\ ${ }^{1}$ Leibniz Institute for Solid State and Material Research Dresden, Helmholtzstrasse. 20, 01069 \\ Dresden, Germany \\ ${ }^{2}$ Karlsruhe Institute of Technology, Hermann-von-Helmholtz Platz 1, 76344 Eggenstein- \\ Leopoldshafen, Germany
}

\section{Introduction}

Over the last two decades, electron tomography (ET), the combination of tomographic methods and transmission electron microscopy (TEM), has considerably contributed to provide new insights into the three-dimensional structure of nanoscale materials [1-5]. In particular, emerging advances in nanoscience are inevitably linked to developments in quantitative two-dimensional (2D) and threedimensional (3D) TEM characterization techniques. In many cases, ET is employed to reconstruct the 3D shape (faceting of crystals) and the distribution or the arrangement (assembly) of nanoparticles down to the nanometer and atomic scale $[1,6-9]$. Moreover, it is used to reconstruct the full 3D morphology of complex nanomaterials and composites [10-13], which can be evaluated further as a basis for quantitative modelling of physical properties [14, 15]. Beyond these capabilities, ET reveals the 3D chemical composition of nanostructures by combining it with spectroscopic methods, such as, electron energy-loss spectroscopy (EELS) and energy-dispersive X-ray spectroscopy (EDS) [16-19]. In specific cases, ET applied together with electron holography enables reconstructing electrostatic potentials in 3D, for example space-charge related diffusion potentials at pn-junctions in semiconductors [20, 21]. In ferromagnetic materials, this approach also allows for the 3D reconstruction of the internal remanent magnetic induction (B-field) [22, 23].

\section{Principle and Practical Aspects}

In order to perform conventional electron tomography on nanoscale materials, a tilt series of projection images is acquired by tilting the sample with the electron microscope goniometer over a large tilt angle range (ideally $180^{\circ}$ ), with a tilt increment of typically $1^{\circ}$ to $3^{\circ}$ (Figure 1a). To this end, special specimen holders with suitable geometry and size are required. Furthermore, software packages for (semi-)automated acquisition are extremely helpful to reduce the total acquisition time and the accumulated electron dose on the sample. Since today's TEM goniometers are not capable to position the specimen with (sub-)nanometer accuracy, the tilt series needs to be aligned in the computer subsequently, i.e., corrected for displacements of the projections with respect to a common tilt axis, e.g., by cross-correlation or fiducial marker alignment. Finally, the aligned tilt series is reconstructed (back-projected) in 3D using a proper mathematical algorithm, such as weighted backprojection technique [24], simultaneous iterative reconstruction technique (SIRT) [25], or more elaborate algorithms [26-31] (Figure 1b). 


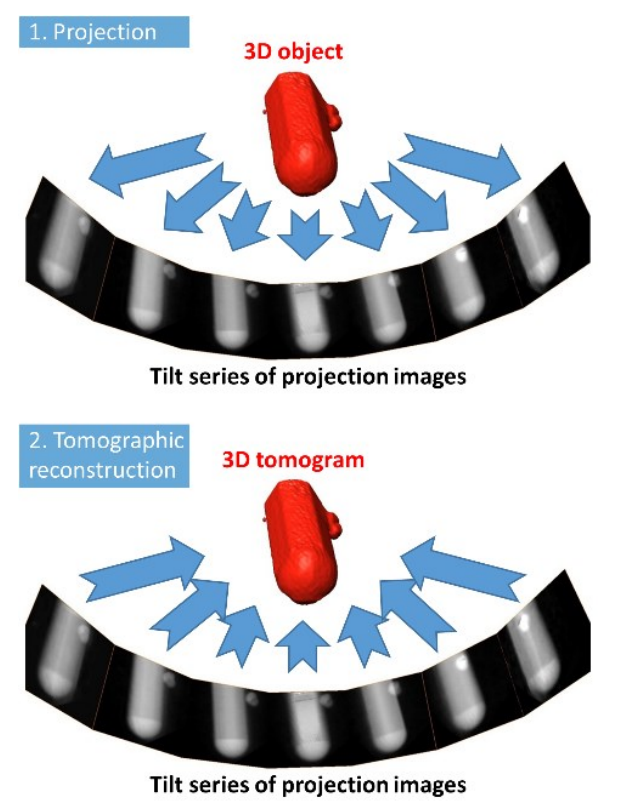

Figure 1. Electron Tomography Principle. In a first step, TEM projections of the 3D object to be investigated are recorded covering as many orientations as possible (ideally in a tilt range of $180^{\circ}$ ) by tilting the sample in the electron microscope. In a second step, the tilt series of projection images serves as input for tomographic reconstruction techniques yielding the $3 D$ tomogram.

\section{Applications}

A variety of TEM imaging modes may be exploited for ET gathering different object properties in 3D and with (sub-)nanometer resolution. The signal detected in the electron microscope has to fulfill the so-called projection requirement for tomography [32], which states that the relation between recorded signal and projected physical property can be expressed by a bijective mapping (monotonic function). In the following, we briefly address three TEM techniques: Bright-field (BF)-TEM (Sect. 3.1), high angle annular dark-field scanning TEM (HAADF-STEM) (Sect. 3.2), and off-axis electron holography (Sect. 3.4) that are suited for 3D electron microscopy. Furthermore, we demonstrate how the 3D data obtained by ET are analyzed to directly link the 3D structure to physical properties of the material (Sect. 3.3).

\subsection{Bright-Field TEM Tomography}

BF-TEM is based on scattering absorption contrast that can be described, in the absence of coherent scattering (dynamical diffraction contrast), as Lambert-Beer type exponential damping of the initial electron beam intensity with increasing sample thickness [33]. The strength of this attenuation is given by a material dependent mean-free-path length (MFPL) of the electron beam transmitting the sample. Thus, the BF-TEM signal is suitable for ET to reconstruct in 3D material contrast and morphology. Figure 2 shows an example of BFTEM tomography applied on self-assembled closely packed spherical Ag nanoparticles (NPs) confined in polymer nanofibers [34]. From a single BF-TEM image (Figure 2a), it is not possible to deduce the helically arranged $\mathrm{Ag}$ nanoparticles as reconstructed in 3D by ET (Figure 2b). 
a)

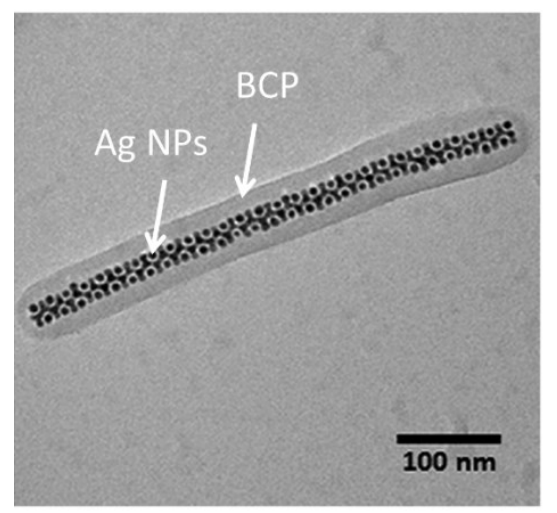

b)

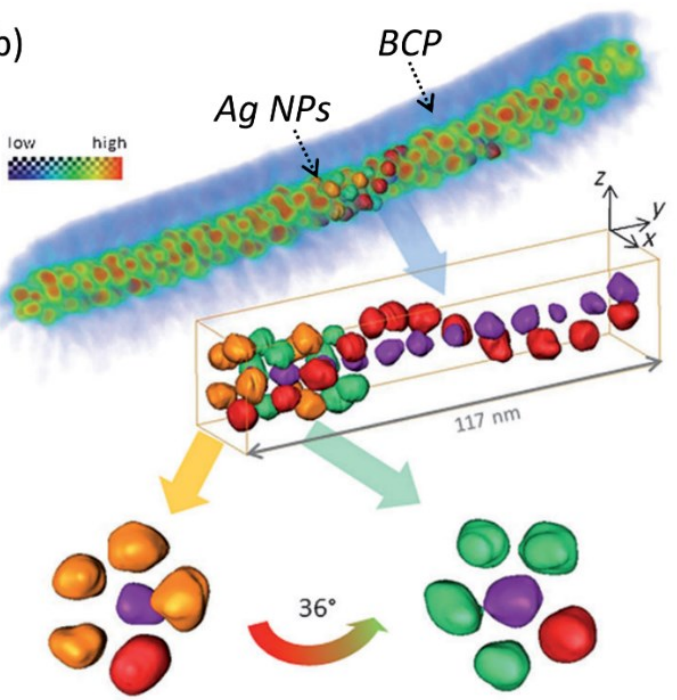

Figure 2. 3D reconstruction of Ag nanoparticle (NP) packing inside the nanofiber (NF) formed by a block copolymer (BCP). (a) TEM image of the NF filled with Ag NPs. The closely packed ordered structure of Ag-NPs is clearly visible. (b) The 3D volume rendering shows the entire $3 D$ structure, in which the reconstructed density is color-coded according to the colorbar, i.e., the Ag-NPs appear red (high density) and the BCP appears blue (low density). Some of the Ag-NPs are segmented to visualize their helical arrangement. This Figure is adapted from Ref.[34].

\subsection{High Angle Annular Dark-Field STEM Tomography}

In materials science, HAADF-STEM is very commonly used as imaging mode for ET $[1,3,5,35]$. This is mainly because the HAADF-STEM signal provides atomic number (Z)-contrast. Furthermore, it is more robust against diffraction contrast compared to the BF-TEM signal. Figure $\mathbf{3}$ depicts the 3D reconstruction of a GaAs/AlGaAs core-multishell nanowire (NW) grown by metalorganic vapor phase epitaxy (MOVPE) using an Au nanoparticle (NP) as catalyst [36]. Such NWs are promising candidates for novel coherent nanoscale light sources (lasers), because they provide an effective gain medium, low-loss optical waveguiding, and strong optical confinement for axially guided optical modes. The Zcontrast allows discriminating between GaAs and AlGaAs within the NW. The cross-section (c) averaged over a well-defined position and thickness (as shown in b) reveals not only the GaAs core and the AIGaAs shell, but also a ca. $5 \mathrm{~nm}$ thin GaAs shell within the AlGaAs, which acts as a quantum well tube (QWT).

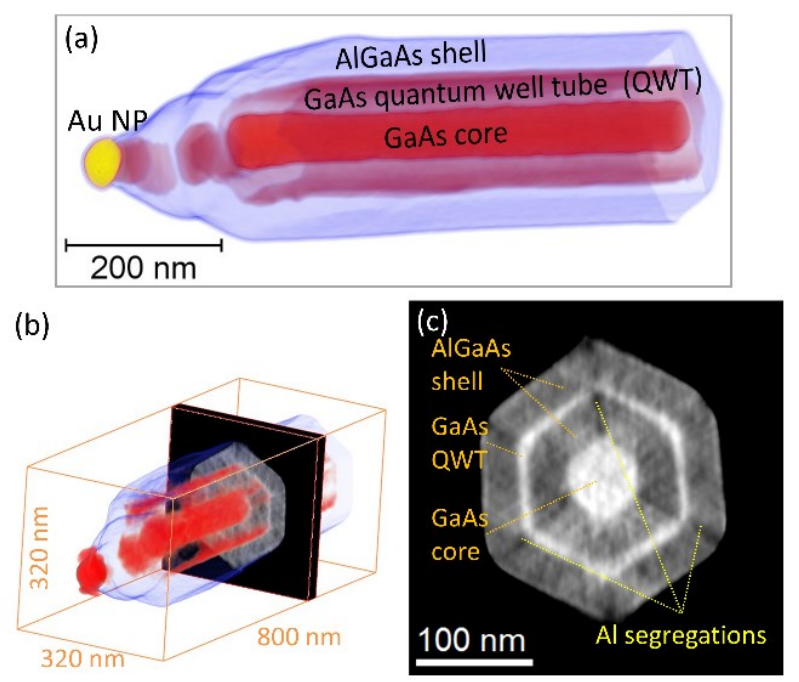

Figure 3. 3D reconstruction by HAADF-STEM tomography of an individual GaAs-AlGaAs core-multishell NW grown along <111> orientation using gold catalyst assisted MOVPE. (a) 3D rendering of the segmented NW structure. (b) Volume rendering of the STEM tomogram with position of the NW cross-section (c) displayed. This Figure is adapted from Ref. [36]. 


\subsection{Linking electron tomography and modeling of physical properties}

Most commonly, ET is employed to offer a semi-quantitative view of the 3D shape or morphology of complex objects. However, recently it is enhanced for a fully quantitative analysis of the structure and properties of materials, e.g. to determine the crystallography of self-assembled nanoparticles $[6,9]$ or to quantitatively describe the 3D morphology of porous media in terms of topology (connectivity \& tortuosity) and geometry (size distribution) [37, 38]. Using ET reconstructions as basis for materials modeling, it is further possible to directly link the structure to physical properties of the material. For example, by analyzing three isomorphic porous silica, it was possible to develop a quantitative description of hindered diffusion for finite sized tracers within the porous network (Figure 4) [14], which is critical to understand e.g. selectivity in catalysis or seperation properties in high performance liquid chromatography (HPLC).
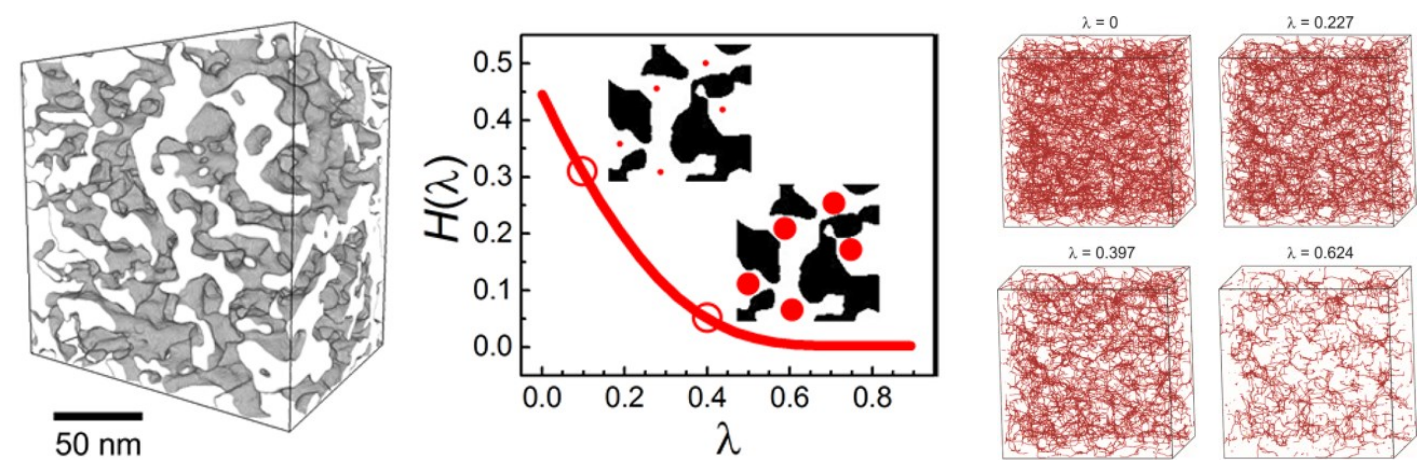

Figure 4. 3D reconstruction by HAADF-STEM tomography of a disordered mesoporous silica. (a) Surface rendering of the segmented pore network. (b) Simulated hindrance factor $H$ for diffusion of a tracer of size $\lambda$ within the pore network. (c) Visualization of the accessible pore network for different-sized tracers. Reprinted with permission from Ref. [14]. Copyright 2018 American Chemical Society

\subsection{Holographic Tomography}

Off-axis electron holography (EH) is an interferometric TEM technique that records the phase shift of an electron wave transmitted through the sample of interest. Since this phase information is proportional to the projections of electrostatic and magnetic potentials, it permits both physical properties to be mapped quantitatively with high spatial resolution and, when combined with tomography to electron holographic tomography (EHT) in 3D [4, 21, 31, 39]. Tomograms obtained by EHT provide the 3D mean inner potential (MIP) distribution from which the 3D morphology and the chemical composition can be inferred [31]. Moreover, functional potentials, e.g., introduced by doping of impurities in semiconductors, can be reconstructed in 3D [21,39]. Figure 5 comprises two recent EHT observations revealing the magnetic B-field within a Co NW [22] and a $\mathrm{CO}_{2} \mathrm{FeGa}$ Heusler alloy NW [23] both with spatial resolution better than $10 \mathrm{~nm}$. The reconstructions of the dominant axial component of the magnetic induction exhibit a small inversion domain at the apex of the Co NW, whereas at the $\mathrm{Co}_{2} \mathrm{FeGa} \mathrm{NW}$, a magnetic dead layer of $10 \mathrm{~nm}$ width was observed. Ongoing work in the field of EHT aims to reconstruct not only one component as presented here, but also all three vector components of the magnetic B-field. This will open new pathways to the characterization of nanoscale ferromagnetic elements, such as spin valves, read-write magnetic heads, magnetic sensors, and magnetic logic devices. 
(a)

(c)

Cobalt Nanowire

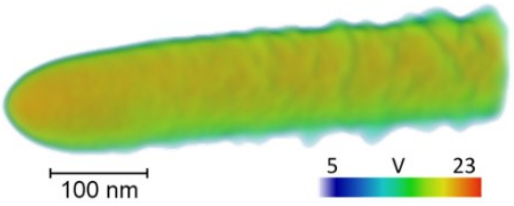

(e)

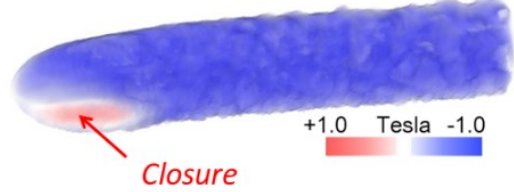

domain

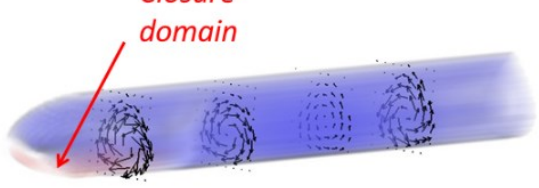

(b)

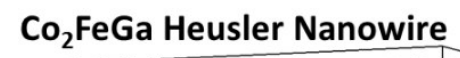

(d)
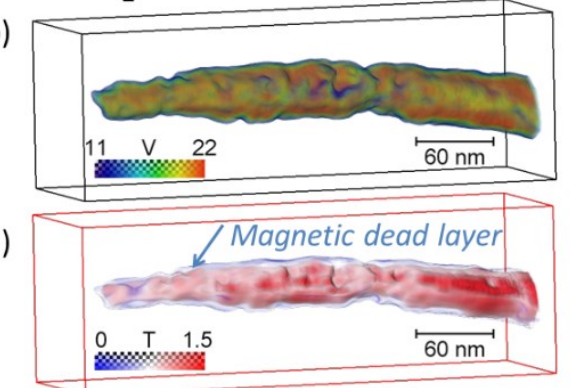

(f)

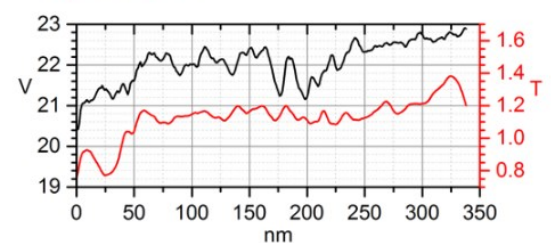

Figure 5. 3D reconstruction of magnetic nanowires (NWs). 3D Volume rendering of electric potential $(a, b)$ and axial (predominant) B-field component (c,d) inside the NWs. Colors correspond to the potential/B-field values. (e) Axial B-field component inside the Co NW obtained from micromagnetic simulation. The arrow plots visualize the out-of-plane components showing the twist of magnetic induction. (f) Line scans in axial direction through the center of the $\mathrm{Co}_{2} \mathrm{FeGa} \mathrm{NW}$ from the tip to the back. This Figure is adapted from Refs. [22, 23].

\section{Conclusion and Outlook}

Electron tomography overcomes the problems of 2D TEM, such as uncertainties in sample orientation, thickness and structure modulations in projection (electron beam) direction, by its capability to reconstruct the full 3D material structure with (sub-)nanometer resolution. Therefore, it is used for the 3D characterization of a large variety of nanostructures, such as nanoparticles, nanowires, complex compound materials, and porous materials. However, in many cases the application of ET is avoided due to the extra amount of time and effort. This might be occasionally caused by the lack of proper and easy-to-use software packages taking over the automated tilt series acquisition and alignment. More importantly, in order to retrieve useful physical 3D information out of the electron tomograms, the latter require to be processed further by demanding and extensive quantification/segmentation steps. Performing these steps in an automated manner is however difficult, because of numerous challenges (e.g., data set variety, low and non-uniform contrast, interfering structures, noise) [40]. Combining modern machine learning models, computer vision techniques and human knowledge for 3D image analysis [41] may overcome these problems and foster ET-based investigations in the field of material science. Recent developments in ET at atomic resolution exist (e.g. Ref. [7, 42]), but a quantitative $3 \mathrm{D}$ reconstruction of individual atoms remains extremely challenging. One route to achieve this goal is to exploit the wave nature of electrons in TEM by performing EHT at atomic resolution [43].

\section{Acknowledgement}

DW acknowledges funding from the European Research Council via the ERC-2016-STG starting grant ATOM.

\section{References}

[1] P.A. Midgley, M. Weyland, Ultramicroscopy 96 (2003) 413.

[2] C. Kubel, et al., Microsc Microanal 11 (2005) 378.

[3] P.A. Midgley, R.E. Dunin-Borkowski, Nat Mater 8 (2009) 271.

[4] D. Wolf, et al., Current Opinion in Solid State and Materials Science 17 (2013) 126.

[5] S. Bals, et al., Angew Chem Int Ed Engl 53 (2014) 10600. 
[6] H. Friedrich, et al., Nano Letters 9 (2009) 2719.

[7] B. Goris, et al., Nat Mater 11 (2012) 930.

[8] C. Ma, et al., Angew Chem Int Ed Engl 54 (2015) 15699.

[9] T.I. Levchenko, et al., Chemistry of Materials 27 (2015) 3666.

[10] T. Kaneko, et al., Macromolecular Symposia 242 (2006) 80.

[11] N. Kawase, et al., Ultramicroscopy 107 (2007) 8.

[12] K. Gries, et al., Ultramicroscopy 109 (2009) 230.

[13] J.S. Barnard, et al., Philosophical Magazine 90 (2010) 4711.

[14] S.-J. Reich, et al., Industrial \& Engineering Chemistry Research 57 (2018) 3031.

[15] G. Haberfehlner, et al., Nano Lett 17 (2017) 6773.

[16] G. Haberfehlner, et al., Nanoscale 6 (2014) 14563.

[17] M. Pfannmoller, et al., Nano Lett 15 (2015) 6634.

[18] B. Goris, et al., Ultramicroscopy 171 (2016) 55.

[19] T.J.A. Slater, et al., Ultramicroscopy 162 (2016) 61.

[20] D. Wolf, et al., Applied Physics Letters 103 (2013) 264104.

[21] D. Wolf, et al., Journal of Physics D: Applied Physics 49 (2016) 364004.

[22] D. Wolf, et al., Chem Mater 27 (2015) 6771.

[23] P. Simon, et al., Nano Lett 16 (2016) 114.

[24] B.K. Vainshtein, Soviet Physics - Crystallography 15 (5) (1970) 781.

[25] P.F.C. Gilbert, Journal of Theoretical Biology 36 (1972) 105.

[26] K.J. Batenburg, et al., Ultramicroscopy 109 (2009) 730.

[27] T. Roelandts, et al., Ultramicroscopy 114 (2012) 96.

[28] B. Goris, et al., Ultramicroscopy 113 (2012) 120.

[29] R. Leary, et al., Ultramicroscopy 131 (2013) 70.

[30] D. Wolf, et al., Ultramicroscopy 136 (2014) 15.

[31] A. Lubk, et al., Physical Review B 90 (2014) 125404.

[32] P.W. Hawkes, "The Electron Microscope as a Structure Projector", in: J. Frank (Ed.) Electron Tomography - Methods for Three-Dimensional Visualization of Structures in the Cell Springer, 2006, pp. 83.

[33] A. Lubk, et al., Applied Physics Letters 105 (2014) 173101.

[34] S. Sanwaria, et al., Angew Chem Int Ed Engl 53 (2014) 9090.

[35] P. Ercius, et al., Adv Mater 27 (2015) 5638.

[36] D. Wolf, et al., submitted (2018).

[37] D. Stoeckel, et al., Langmuir 30 (2014) 9022.

[38] D. Stoeckel, et al., Langmuir 31 (2015) 7391.

[39] A.C. Twitchett-Harrison, et al., Nano Letters 7 (2007) 2020.

[40] K. Sandberg, 79 (2007) 769.

[41] I. Luengo, et al., J Struct Biol 198 (2017) 43.

[42] M.C. Scott, et al., Nature 483 (2012) 444.

[43] J. Krehl, "Incorporating Fresnel-Propagation into Electron Holographic Tomography - A possible way towards three-dimensional atomic resolution", Technische Universität Dresden, 2017. 